\title{
A REVIEW OF OPERATOR THEORY IN QUANTUM MECHANICS: A CASE OF MICROWAVES, ELECTRICITY AND MAGNETISM
}

\author{
PAUL G. KHUMBA, N. B. OKELO
}

Department of Mathematics, Malawi University of Science and Technology

Email: khumba@yahoo.com

School of Mathematics and Actuarial Science, Jaramogi Oginga Odinga University of Science and Technology, P. O. Box 210-40601, Bondo-Kenya

Email: bnyaare@yahoo.com

Keyword: Microwaves, Wavelength, Magnetic field

\section{ABSTRACT}

We review the significance and input of operator theory in the field of quantu nechanic In particular, we survey the world of microwaves. We also explore the applicarons in tricit and magnetism.

\section{Introduction}

Faraday was the founder of the existing knowledge concerning leck ty and masetism. Maxwell, the mathematician and his contemporary, was the first was w articip in the description of the electric phenomena and processes and the magnetic ones, which we observed by Faraday. Maxwell obtained the equations, which are considere to solve all the problems connected with electricity and magnetism still [1].

$$
\operatorname{rot} \bar{E}=-\frac{1}{C} \frac{\partial \bar{B}}{\partial t} \text {, }
$$

Here: $\bar{E}=\bar{E}(\bar{r}, t)$ is electric field strenoth;

$$
\operatorname{div} \bar{E}=4 \pi \rho,
$$

$\bar{B}=\bar{B}(\bar{r}, t)$ is magnetic fie

$$
\operatorname{rot} \bar{B}=\frac{1}{C} \frac{\partial \bar{E}}{\partial t}+\frac{4 \pi}{C} \bar{Y}
$$
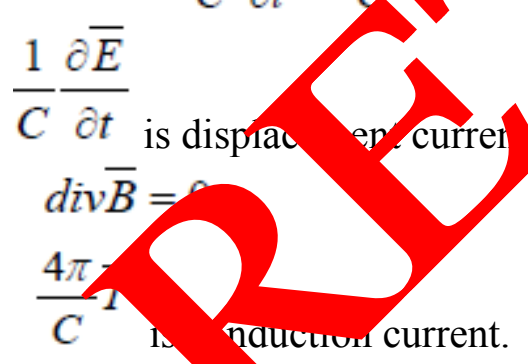

It is consid that Maxwell equations describe a propagation of the so-called electromagnetic waves. A mod 11 of Maxwell electromagnetic wave is given in Fig. 1 [1].

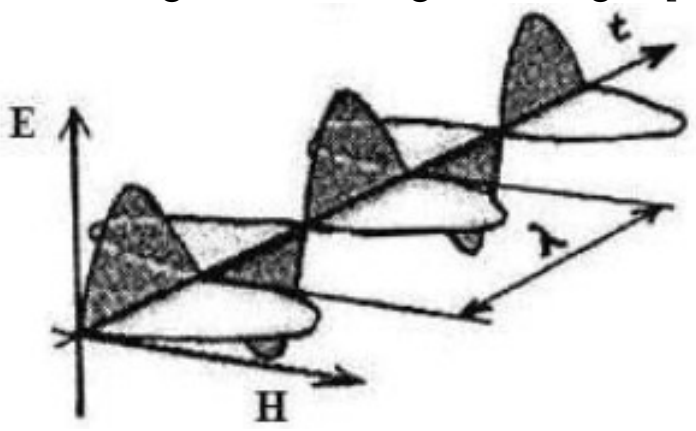

Fig. 1. Diagram of the electromagnetic wave 
As it is clear, Maxwell equations (1-4) are represented in partial derivatives. Partial derivatives are taken according to time and a coordinate usually, but as these both variables are changed synchronously in the implementation of any process, the mathematicians have decided to leave one of the parameters (for examples, time $t$ ) unchanged and to differentiate with respect to the coordinate $\bar{r}$, the second parameter, without further ado in order to facilitate a gain of the equations, which describe this process. But it does not take place in reality. In reality, the coordinate $\bar{r}$ and time $t$ are changed simultaneously; that's why one cannot leave the coordinate $\bar{r}$ constant when the equation is differentiated with respect to time $t$ and one cannot stop time $t$ when the equation is differentiated with respect to the coordinate $\bar{r}$. Such process does not exist in reality, because time cannot be stopped, and the nonpermanent coordinate is always time function [1].

Thus, an elementary analysis of Maxwell equations shows that the rules, which dicort reà are laid in the process of their solution as time cannot be stopped and the rdinate o the displacement of any object in space is always time function. We see that Mavell ations a e at variance with the procedure of their acquisition and solution, but we cannclexpress $h$ sse of this contradiction clearly. A question arises: do Maxwell equa ns df ribe dity or mysticism?[1].

Surely, the approximate methods of the solution of Maxwell a ons can $\mathrm{g}$ a result, which coincides with the experiment. The essence of this coinciden is in fact that the approximate methods of the solution of Maxwell equations are based crose of Fo /series. This method is used when the results of in the experimental data are $\mathrm{p}$ ocessed. It means that the physical essence of the electromagnetic wave is not given in the proce of the exp rimental data processing. This wave can have various physical fillings, which are no egistered by the measuring instruments. Under such conditions, a coincidence of the experimen. with the theoretical one can be fortuitous, and its interpretation can be comp arroneous. The fact that Maxwell equations describe only a part $\left(10^{-4} \ldots 10^{6} \mathrm{~m}\right)$ of the wb $/ 0$ ale $\left(10^{-18} \ldots 10^{6} \mathrm{~m}\right)$ of the so-called electromagnetic radiation (Table 1), and they fa to describe its remaining part $\left(10^{-4} \ldots 10^{-18} \mathrm{~m}\right)$. Why? The answer is given below.

Table 1. Bands of changes on wavengths $\lambda$ ) and masses of electromagnetic radiations

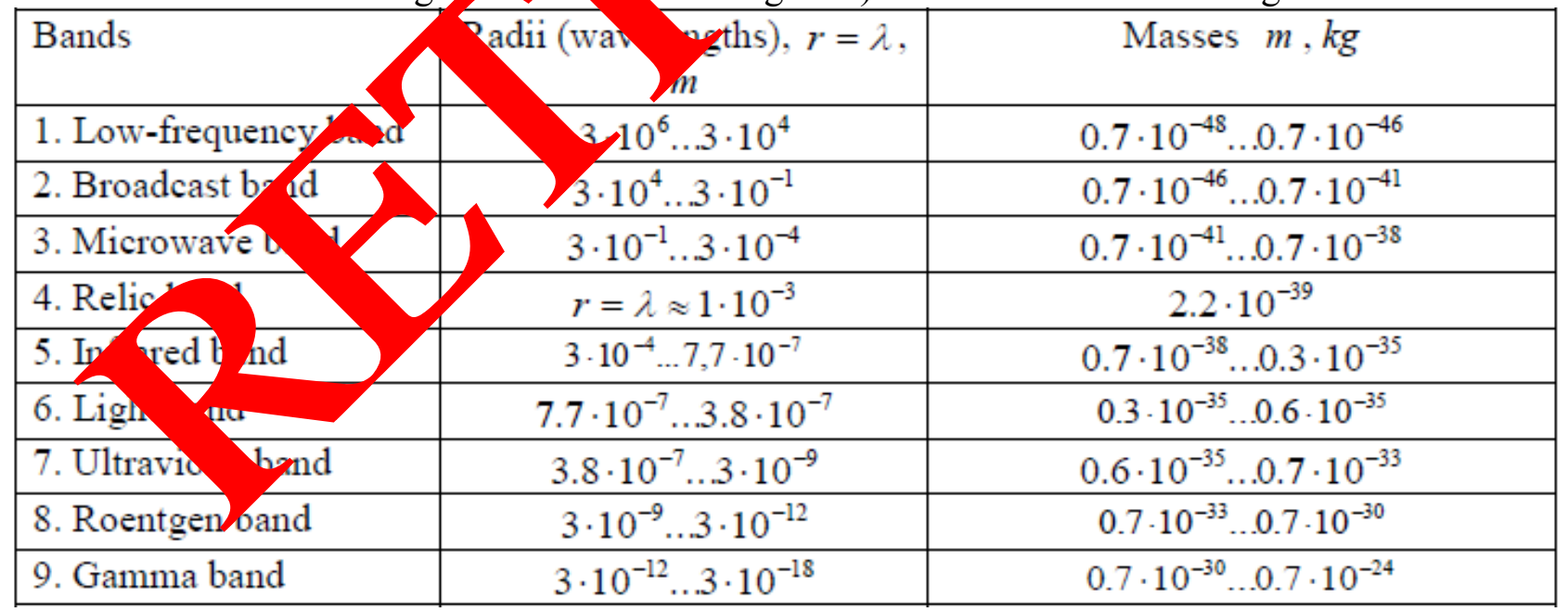

Let us pay attentions to Louis de Broglie famous wave equation [1]

$$
y=A \sin 2 \pi(v t-x / \lambda) \text {. }
$$

The mathematical symbols, which make this equation, are well known: $\lambda$ is wavelength, is wave frequency, $t$ is time, $x$ is the coordinate. It is clear that the coordinate $x$ and time $t$ in the equation (5) in the same way as in Maxwell equations (1-4) are the independent variables. It is at variance with the reality. Where has such contradictory equation, which describes reality supposedly, come from? 
The ideas concerning the wave nature of various phenomena were easily recognized as the authentic ones, because they result from the observed formation and propagation of the waves on the water surface (Fig. 2) [1].

Let us suppose that an ocean wave has propagated at the distance of $1000 \mathrm{~km}$. Does the equation (5) make it possible to determine time, which is required for this wave to propagate at the distance of $1000 \mathrm{~km}$ ? There is no answer. Let us put the next question: at what distance will the wave be in a minute after its formation? There is no answer again. Why? The answer is clear. In this equation, the coordinate $x$ and time $t$ are independent variables. Let us take another approach to this equation and put the next question: at what moment beginning from the wave formation has its amplitude become equal to $+Y A$ ? The equation (5) gives us innumerable quantity of the distances amplitude has the same value $+Y A$. Let us put the next question: why does not I culs de glie equation (5) allow us to determine the coordinate $x$ of the wave at the specified stant in ti $t$ ? The answer is obvious. It is because the equation (5) makes it possible to deternine $\mathrm{v}$ an ord hate of the wave, to which a large quantity of the coordinates $x i$ corresponds $\mathrm{f} / \mathrm{m}$ the bes ing of its formation; in these coordinates, the ordinate $-Y i$, in which we are inte sted $i$ bas o, and the same value [1].

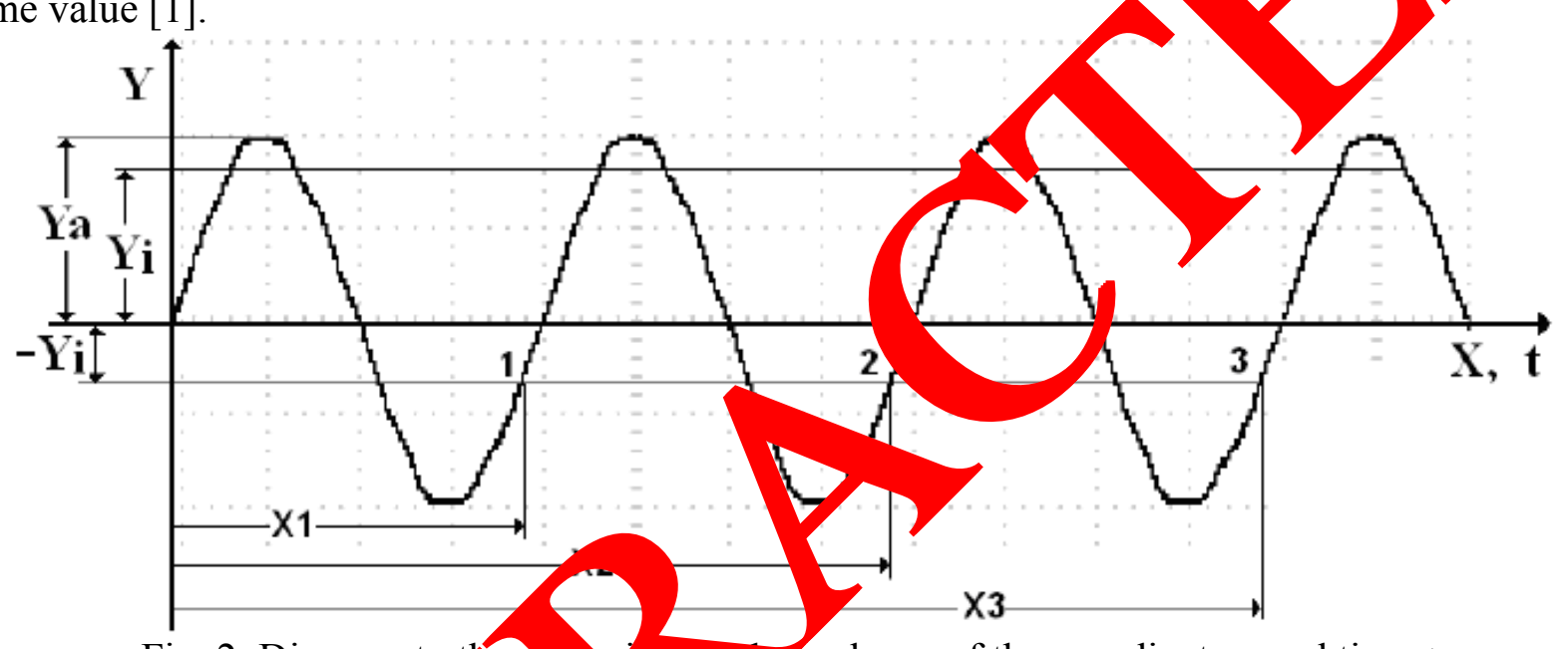

Fig. 2. Diagram to thendence of the coordinate $x$ and time $t$

Now, we have come to a nain ques can Louis de Broglie equation (5) be used for a description of the elect magn wave motion? The answer is univocal: no, it cannot, because we shall fail to deter allne the nu. $y$ of the propagating waves (it is called a wave packet) theoretically; it neans at we cannot know the coordinates of the center of this packet at any instant in time. $\mathrm{n}$ out th a result of our analysis resembles a guess-work [1].

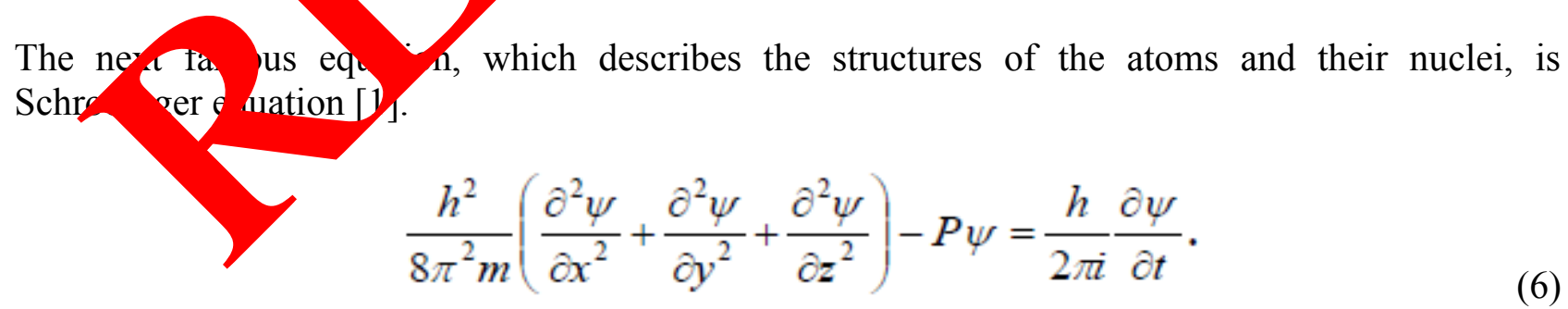

It is easy to see that the coordinates and time are independent variables in this equation (6) as well; it is at variance with the reality where the coordinate is always a time function. Nevertheless, orthodox people are proud of the fact that the old theory of microworld describes the structures of its inhabitants with the help of Schrodinger equation, which makes it possible to determine only a probabilistic position of the electron in the atom and the elementary particles in its nucleus. Thus Schrodinger equation describes the position of the electrons in the atoms (Fig. 3) [1]. 

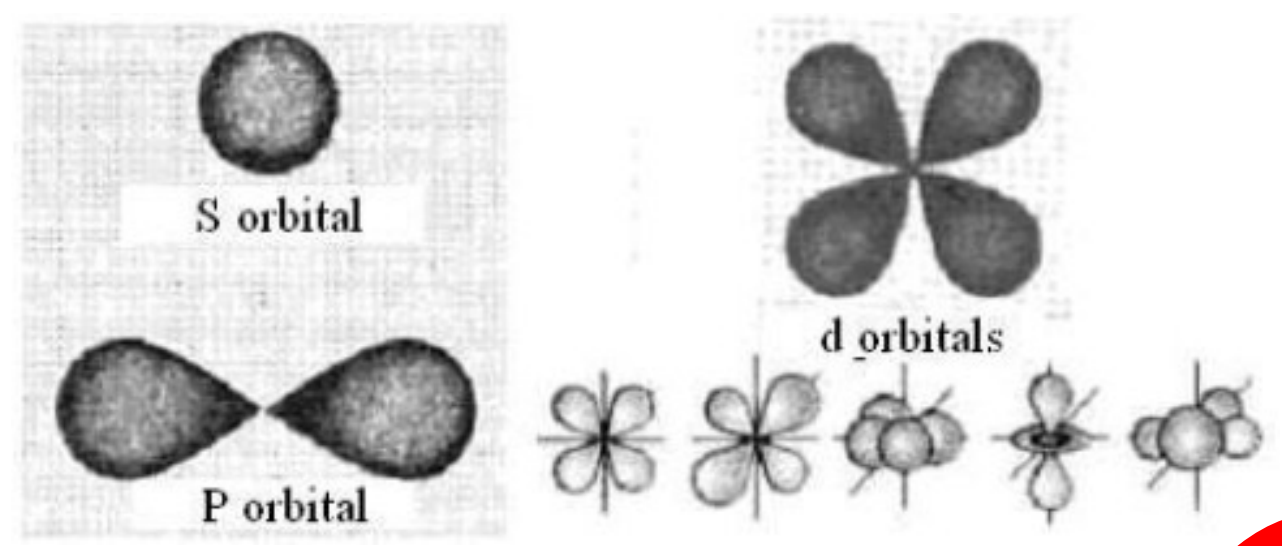

Fig. 3. Forms of the electron clouds

As it is clear (Fig. 3), the forms of the electron clouds have little in comm $0 \mathrm{n}$ with the ms of the orbits; that's why the chemists have called them the orbitals without ther In s. 4 , the models of the atoms resulting from Schrodinger equation (6) are giver 9 ].

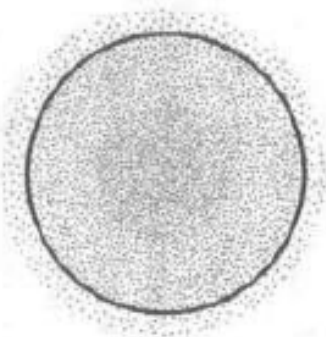

Atom of hydrogen

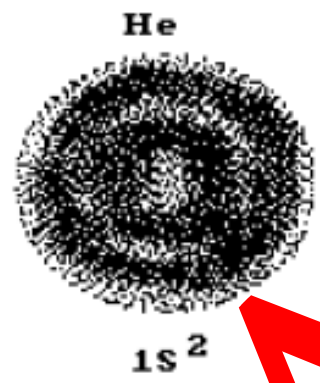

Atom of helium

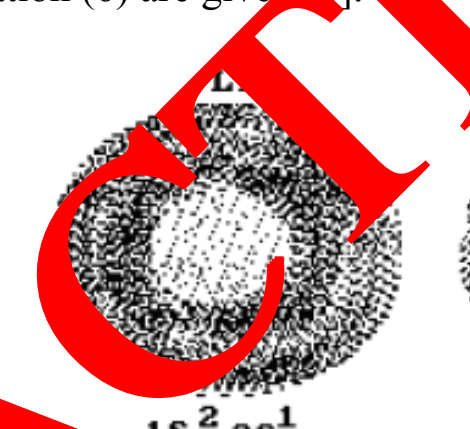

$1 \mathrm{~s}^{2} 2 \mathrm{~s}^{1}$

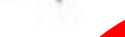

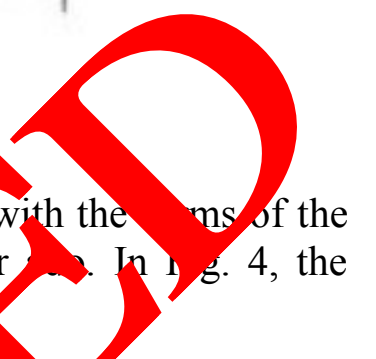

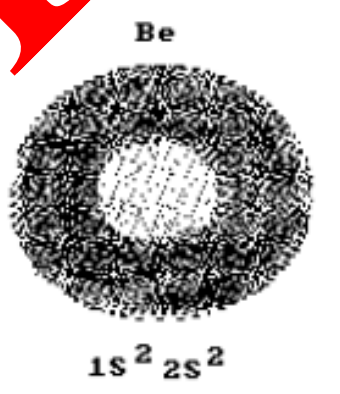

Atom of beryllium

Fig. 4. Models of the atoms drogen, helium, lithium and beryllium resulting from the old heory microu $1 \mathrm{~d}$, Schrodinger equation

In Fig. 5, a, the hydrogen morecul odel, which results from the old theory of microworld, Schrodinger equation, is of in Fig.s, the hydrogen molecule model, which results from the new theory of microwo $\mathrm{d}$, is a n [1].

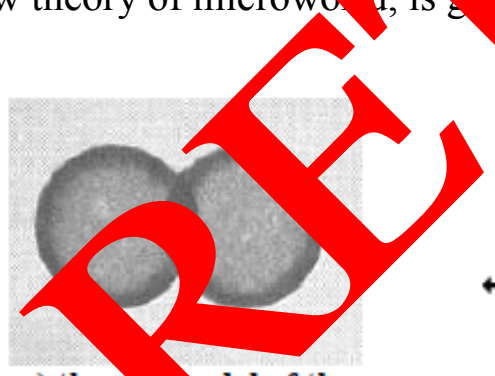

a) the or of the hydrogen mole, with the covalenthoond

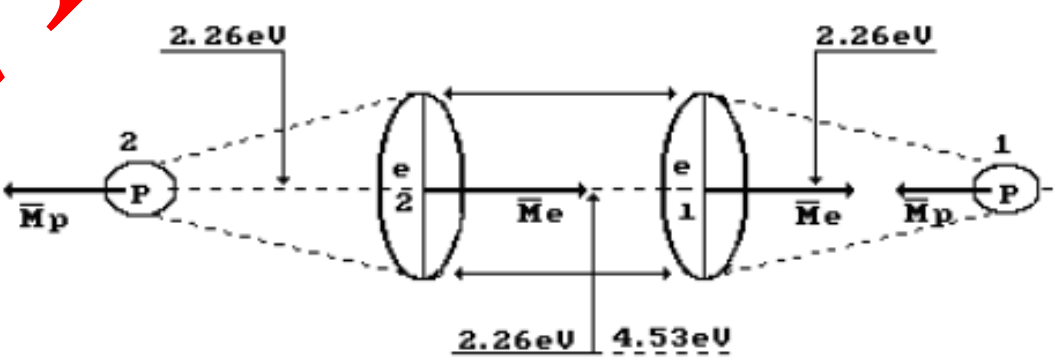

b) the new model of the hydrogen molecule with the covalent bond

Fig. 5. The old and the new models of the hydrogen molecule with the covalent bond

The latest achievements of the old theory of microworld in the discovery of the structures of the atomic nuclei are given in Fig. 6 [2]. As the author notes, the dark spots and the bright ones on the nuclei rings are the intermittent protons and the neutrons [2]. The way, in which the rings are interconnected as well as the protons and the neutrons in these rings, is a sealed book. Nevertheless, it seems to satisfy the orthodox people [2]. 


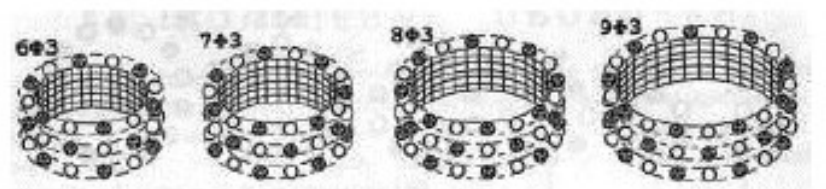

a)

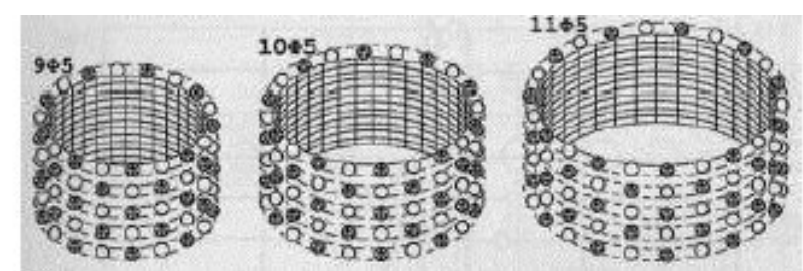

b)

Fig. 6. External ring shells of the atom nuclei

The astonishing results originate from the probabilistic Schrodinger equation. The atoms (Fig. 4) are the misty spots; the nuclei (Fig. 6) are the rings of the unknown nature, which hold the intermittent protons and neutrons with an inexplicable bond between them. It is a and nothing more.

\section{RESULTS}

Where is reality? As global errors are abundant, there is the only way we s.ald rey rn the analysis of the initial notions and axioms, which serve as a basis for hu achiev hents in the perception of surrounding environment, and check completeness f the axio wich lay in the foundation of the existing knowledge, which has become cover a th many con adictions [1].

From the analysis being described, the contradictions esur, incomp nsion of the criterion significance of the connection between space, matter a dime, the primary elements of creation, is their main cause. The philosophers discuss the philoso ical essenc of the space, matter and time unity long ago; but the physics-theoreticians fail to pay ntion to he criterion significance of this unity in the evaluation of authenticity of the rocesses description of the material object motion in space [1].

Space, matter and time unity points or the fact hat an interconnection between matter, space and time should be reflected in all $\mathrm{m}$ them cal mod $1 \mathrm{~g}$, which describe changing reality. This rule, which is quite simple by sigh, $y$ moined annoticed neither by the mathematicians, nor physicists [1].

All phenomena and proce in Naturo koplace within the framework of the Unity Axiom. The processes of the disp' cem of any object in space are inseparable from time flow. All displacements are tir tunction we ignore this fact, we shall get an approximate or completely distorted notion oncer ing a phe menon or a process being studied as it results from Maxwell equations, Loy Bre one equation and Schrodinger equation [1].

Let us p ntion the act that the mill the of the place thin $t$ framew $k$ of the Unity Axiom. It was violated when a transition to the microworld behavio $\quad$ th the help of the wave theories took place. No attention was paid to the fact that all e riments, which were performed by man, took place and take place within the framework of Unity Axiom beyond his will. It is natural that a correct interpretation of the results of these experiments is possible only with the help of the theories and mathematical models, which operate within the framework of the Unity Axiom as well [1].

If we use the mathematical models and theories, which operate outside the framework of the Unity Axiom, for example, equations (1-6), for an interpretation of the experimental results, we shall get an approximate notion concerning the phenomenon being studied at best and a completely distorted notion at the worst [1].

It is known that the notion of the photon was introduced into science later than the notion of electromagnetic radiation; everything, which was connected with the notion of the photon, was 
developed in parallel with the things, which were connected with than the notion of electromagnetic radiations (Table 1). Nobody began to think about the physical essence of the distinguishing features of natural formations, which are present in these notions. We have already analyzed the physical essence, which is present in the notion of electromagnetic radiation. Let us see what is in the notion of the photon?

An analysis of all existing mathematical models, which describe the photons, results in the following magnetic model (Fig. 6, a). It is a formation, which is localized in space, not Maxwell wave (Fig. 1). The equations of the motion of the center of mass $M$ of the photon within the framework of the Unity Axiom, which obliges its coordinates to be the time functions, are as follows [1]:

$$
\begin{aligned}
& x=C t+0.067 r \sin 6 \omega_{0} t \\
& y=0.067 r \cos 6 \omega_{0} t .
\end{aligned}
$$

It results from these equations that the center of mass $M$ of the photon (Fic, a) $c$ mscri es a curtate cycloid with a very small amplitude [1]

$$
A=\rho_{M}=\frac{r}{2}\left(1-\cos \frac{\alpha}{2}\right)=0.067
$$

and average velocity of the center of mass $\mathrm{M}$ of the photon (th $\mathrm{ag}_{\mathrm{h}}$ is change remains constant and equal to $\mathrm{C}$ (Fig. 7, b) [1].

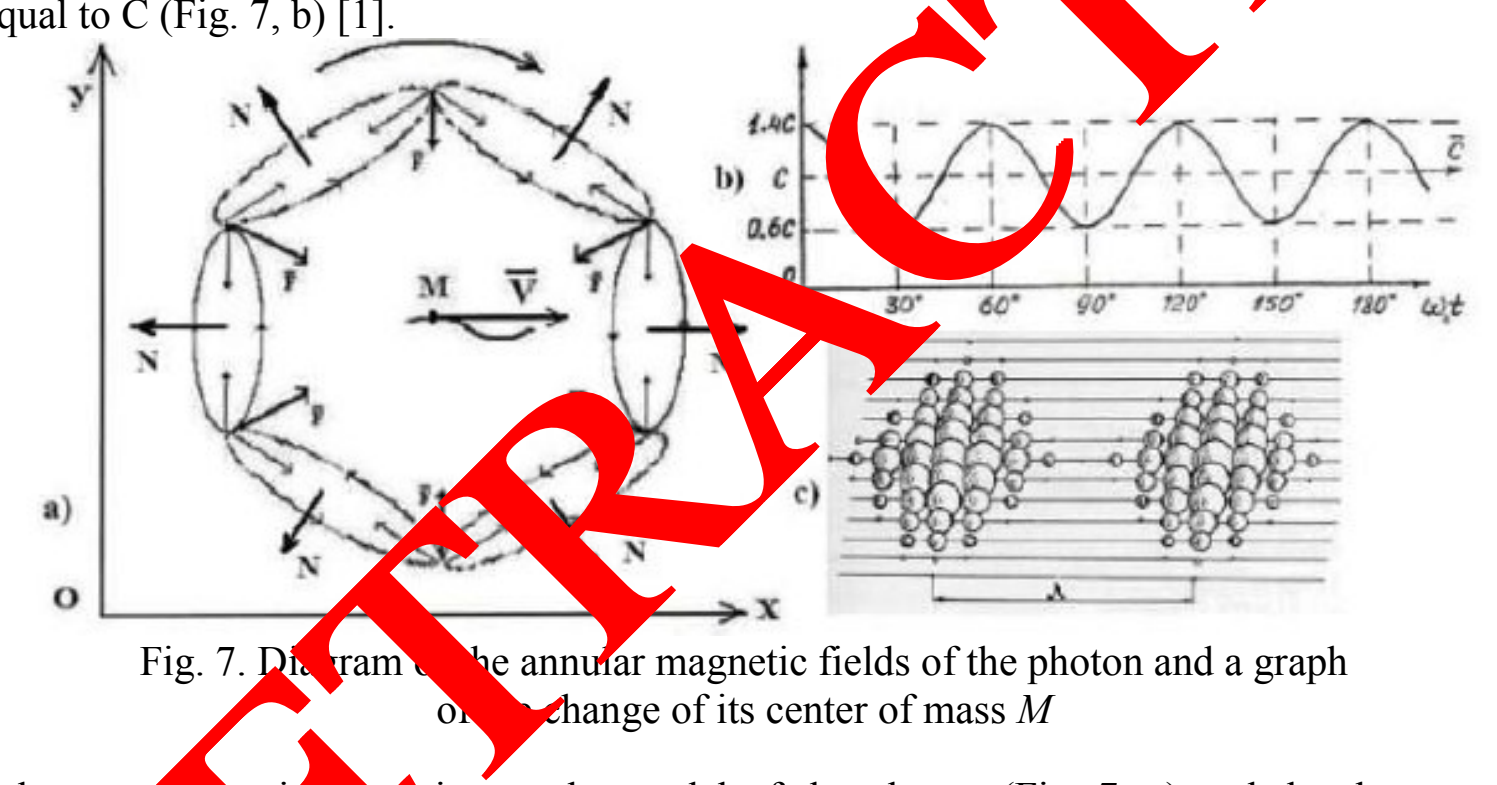

Now, let us pun nai e que tion to the model of the photon (Fig. 7, a) and the theory, which describes it within frame ork of the Unity Axiom. As the photons of all frequencies and radii move in spa with and the same uniform velocity, center-of-mass velocity of the photon shoul ot de end on is radius $r$. Does it result from the equations of motion (7) and (8) of the center 0 photon. The answer is in the following formula of the center of mass $M$ of the photon, wh is derived from the equations (7) and (8) automatically [1].

$$
\begin{gathered}
V_{M}=\sqrt{(d x / d t)^{2}+(d y / d t)^{2}}= \\
=\sqrt{C^{2}+0.85 C^{2} \cos 6 \omega_{0} t+0,18 C^{2}}=\sqrt{\frac{1.18+0.85 \cos 6 \omega_{0} t}{\varepsilon_{0} \cdot \mu_{0}}} .
\end{gathered}
$$

As it is clear (10), center-of-mass $M$ velocity of the photon does not depend on its radius, which is changed in the interval of 15 orders of magnitude $\left(10^{-3} \ldots 10^{-18} \mathrm{~m}\right)$. The rest interval $\left(10^{-3} \ldots 10^{6} \mathrm{~m}\right)$ forms an aggregate of the photons (Fig. 7, c), which are reflected from an aerial and are polarized in the plane of incidence and the reflecting plane and thus orientate the free electrons of the aerial by its resultant polarized field and form electric potential in it; this potential is measured and processed by a receiving device [1]. 
The proton and the neutron, the next important inhabitants of microworld, are given in Fig. 8 [1].

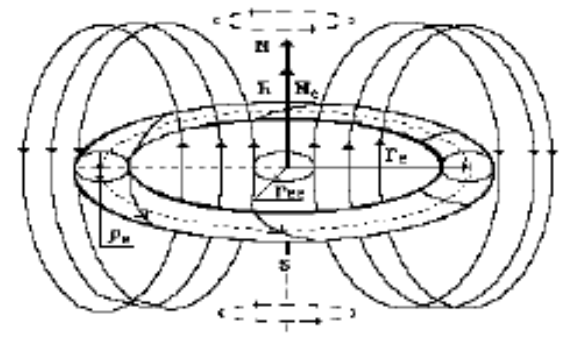

a) model of the electron

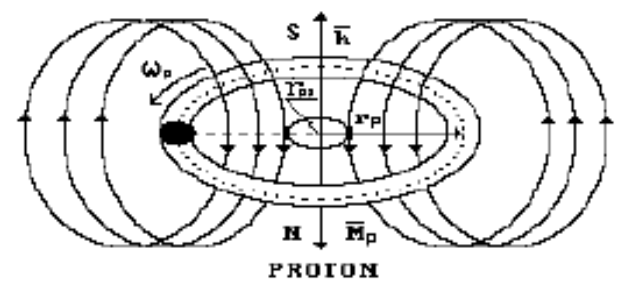

b) model of the proton

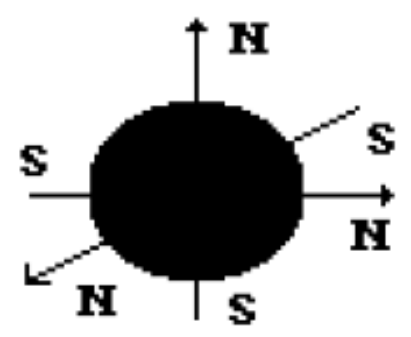

c) model of the neutron

Fig. 8. Diagrams of the structures: a) of the electron; b) of the proton; c) of the $n$

The electron (Fig. 8, a) is a hollow torus with two revolutions: in relation of the tral axis a 1 in relation to the ring axis of the torus. It has two magnetic poles: the north pole and south pole S. The vectors of its spin $\bar{h}$ and magnetic moment $\bar{M}_{e}$ are directed a ong the cen a coincided according to the direction. The proton (Fig. 8, b) is a solid or Tb vect is of its spin $\bar{h}$ and magnetic moment $\bar{M}_{p}$ are directed along the revolution ax as we con ariwise. The neutron has six magnetic poles, which are situated along th 0 dinate ax (Fig. 8, c). The electromagnetic structures of the electric, the proton and the eutro re described by dozens of mathematical models, which include nearly 30 constan she shall s on how the given structures of the proton and the neutron participate in he formation of the structures of the atom nuclei, and the structures of the electron and the $\mathrm{p}$ ton implem ent its functions in the atom formation superbly [1].

The laws, which form the atoms, are hidden in mental spectroscopy, the largest experimental data array. The process of the interaction of the lec 10 the atom nucleus results from it. As the new theory of microworld has the law of forn tion of the spectra of the atoms and the ions and binding energies $E_{b}$ of the electrons with e proto of the nuclei, not only calculates the spectra of all atoms and ions, but discloses sics if the linear interaction of the electrons of the atoms with the protons of their nuclei. T/ the simple form [1]

A mathematical $\mathrm{de}^{\prime}$ of the ormation of binding energies of the electrons with the protons of the nuclei is eyen simp,

$$
2 E_{b}=\frac{E_{i}}{n^{2}}=\frac{E_{1}}{n^{2}} .
$$

The law o rmation of the spectra of the atoms and the ions in case of the interlevel passes of electrons has form, which similar to the orthodox one, but with another physical content of the terms before the brackets [1]

$$
E_{f}=E_{1} \cdot\left[\frac{1}{n_{1}^{2}}-\frac{1}{n_{2}^{2}}\right]
$$

In the given formulas, $E_{f}$ are energies of the emitted or absorbed photons; $E_{i}$ are energies of ionization of the atoms; $E_{1}$ are binding energies of the electrons with the nucleus, which correspond to the first energy levels. The calculation results of the spectra of some atoms according to the given formulas (11) and (12) are specified [1]. 
Table 2. Spectrum of the hydrogen atom spectrum

\begin{tabular}{|l|c|c|c|c|c|c|}
\hline Values & $\mathrm{n}$ & 2 & 3 & 4 & 5 & 6 \\
\hline$E_{f}(\mathrm{exp})$ & $\mathrm{eV}$ & 10.20 & 12.09 & 12.75 & 13.05 & 13.22 \\
\hline$E_{f}$ (theor) & $\mathrm{eV}$ & 10.198 & 12.087 & 12.748 & 13.054 & 13.220 \\
\hline$E_{b}$ (theor) & $\mathrm{eV}$ & 3.40 & 1.51 & 0.85 & 0.54 & 0.38 \\
\hline
\end{tabular}

Table 3. Spectrum of the first electron of the helium atom

\begin{tabular}{|l|c|c|c|c|c|c|}
\hline Values & $\mathrm{n}$ & 2 & 3 & 4 & 5 & 6 \\
\hline$E_{f}(\exp )$ & $\mathrm{eV}$ & 21.22 & 23.09 & 23.74 & 24.04 & 24.21 \\
\hline$E_{f}$ (theor) & $\mathrm{eV}$ & 21.22 & 23.09 & 23.74 & 24.05 & 24. \\
\hline$E_{b}$ (theor) & $\mathrm{eV}$ & 3.37 & 1.50 & 0.84 & 0.54 & 0.37 \\
\hline
\end{tabular}

Table 4. Spectrum of the first electron of the boro

\begin{tabular}{|c|c|c|c|c|c|c|c|}
\hline Values & $\mathrm{n}$ & 2 & 3 & 4 & & 6 & 7 \\
\hline$E_{f}(\exp )$ & $\mathrm{eV}$ & 4.96 & 6.82 & 7.46 & & & 8.02 \\
\hline$E_{f}$ (theor) & $\mathrm{eV}$ & 4.96 & 6.81 & & & 7.93 & 8.02 \\
\hline Values & $\mathrm{n}$ & 8 & 9 & & 11 & 12 & 13 \\
\hline$E_{f}(\exp )$ & $\mathrm{eV}$ & 8.09 & 8.13 & 8.16 & 8.18 & 8.20 & 8.22 \\
\hline$E_{f}$ (theor) & $\mathrm{eV}$ & 8.09 & 8.13 & & 8.18 & 8.20 & 8.22 \\
\hline Values & $\mathrm{n}$ & 14 & & & 17 & 18 & 19 \\
\hline$E_{f}(\exp )$ & $\mathrm{eV}$ & 8.23 & & & 8.25 & 8.26 & $\ldots$ \\
\hline$E_{f}$ (theor) & $\mathrm{eV}$ & & & 8.25 & 8.25 & 8.26 & $\ldots$ \\
\hline
\end{tabular}

Table 5. Sp tru ectron of the beryllium atom

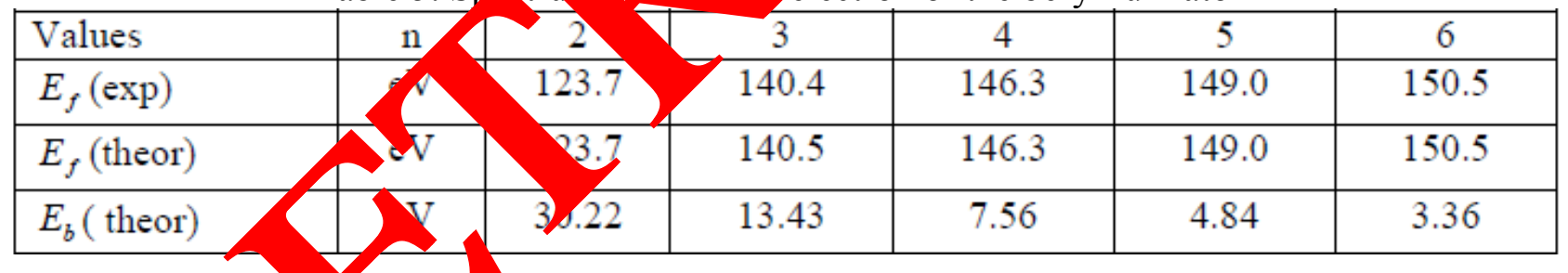

\begin{tabular}{|c|c|c|c|c|c|c|}
\hline & \multicolumn{7}{c}{. Spectrum of the first electron of the carbon atom } \\
\hline & $\mathrm{n}$ & 2 & 3 & 4 & 5 & 6 \\
\hline$E_{f}$ (the & $\mathrm{eV}$ & 7.68 & 9.67 & 10.37 & 10.69 & 10.86 \\
\hline$E_{b}$ (theor) & $\mathrm{eV}$ & 7.70 & 9.68 & 10.38 & 10.71 & 10.88 \\
\hline
\end{tabular}

Table 7. Spectrum of the second electron of the oxygen atom

\begin{tabular}{|l|c|c|c|c|c|c|}
\hline Values & $\mathrm{n}$ & 2 & 3 & 4 & 5 & 6 \\
\hline$E_{f}(\exp )$ & $\mathrm{eV}$ & 14.12 & 25.83 & 29.81 & 31.73 & 32.88 \\
\hline$E_{f}$ (theor) & $\mathrm{eV}$ & 14.12 & 25.79 & 29.87 & 31.76 & 32.78 \\
\hline$E_{b}$ (theor) & $\mathrm{eV}$ & 21.00 & 9.33 & 5.25 & 3.36 & 2.33 \\
\hline
\end{tabular}


Table 8. Binding energies $E_{b}$ of the electron of the hydrogen atom $e_{H}$ and the electrons $(1,2,3,4)$ of the beryllium atom $B_{e}$ with the nucleus at the time when all of them are in the atom

\begin{tabular}{|c|c|c|c|c|c|c|c|c|c|}
\hline $\mathrm{n}$ & 1 & 2 & 3 & 4 & 5 & 6 & 7 & 8 & 9 \\
\hline$e_{H}$ & 13.6 & 3.40 & 1.51 & 0.85 & 0.54 & 0.38 & 0.28 & 0.21 & 0.17 \\
\hline 1 & 16.17 & 4.04 & 1.80 & 1.01 & 0.65 & 0.45 & 0.33 & 0.25 & 0.20 \\
\hline 2 & 16.17 & 4.04 & 1.80 & 1.01 & 0.65 & 0.45 & 0.33 & 0.25 & 0.20 \\
\hline 3 & 16.17 & 4.04 & 1.80 & 1.01 & 0.65 & 0.45 & 0.33 & 0.25 & 0.20 \\
\hline 4 & 16.17 & 4.04 & 1.80 & 1.01 & 0.65 & 0.45 & 0.33 & 0.25 & 0.20 \\
\hline & & & & & & & & & \\
\hline $\mathrm{n}$ & 10 & 11 & 12 & 13 & 14 & 15 & 16 & 17 & 18 \\
\hline$e_{H}$ & 0.14 & 0.11 & 0.09 & 0.08 & 0.07 & 0.06 & 0.05 & 05 & 4 \\
\hline 1 & 0.16 & 0.12 & 0.10 & 0.08 & 0.07 & 0.06 & 0.05 & 0.05 & 0. \\
\hline 2 & 0.16 & 0.12 & 0.10 & 0.08 & 0.07 & 0.06 & 0 & 0.0 & 0 \\
\hline 3 & 0.16 & 0.12 & 0.10 & 0.08 & 0.07 & 0.06 & 0.05 & 0.05 & 0.04 \\
\hline 4 & 0.16 & 0.12 & 0.10 & 0.08 & 0.07 & 0.06 & 5 & 0.0 & 0.04 \\
\hline
\end{tabular}

The laws of formation of the spectra of the atoms and the ions (1/ , (12) a 13$)$ ad Tables 2-8 show that show that there are no energy of orbital motion of the ons in the are only energies of linear interaction of the electrons with the pr tons he nucler. The spectrum of four electrons of the beryllium atom (Table 8) proves a li interact of its electrons with the protons of the nucleus; it proves an arrangement of the protons (white $b ; 1 \mathrm{~s}$ ) on the surface of the nucleus automatically (Fig. 9) [1].

The structure o nu se the beryllium atom (Fig. 9) explains a cause of the existence of its stable nuclei only five $n$ atrons and four protons. With the specified quantity of the neutrons and the $p \quad s$ in $t$ uclus, it can have only such structure, which is shown in Fig. 9. If the proton and th neutron are presented in a simplified way in the form of the spherical formations, it is clear $t \mathrm{t}$ heutron has four contacts with the adjacent neutrons in plane [1].

A symme, structure of the nucleus of the carbon atom, which is in a diamond (Fig. 10, a), proves that the cent Cutron has six contact poles. Other neutrons have the same number of the contact poles, but notall their contacts participate in the interactions [1].

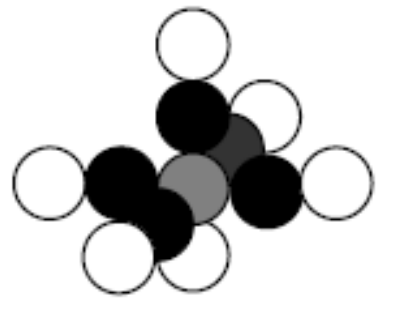

a)

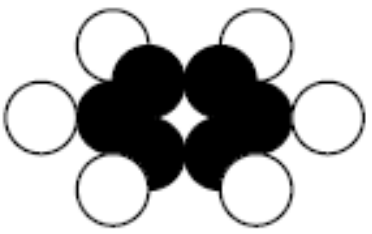

b)

Fig. 10. Structural diagrams of the nucleus of the carbon atom: a) diagram of the spatial nucleus (diamond); b) diagram of the flat nucleus (graphite) 
As the atom electrons interact with the protons of the nuclei linearly, strength of diamond results from the structure of the spatial nucleus of the diamond atom (Fig. 10, a); this strength is stipulated by an ideal spatial symmetry of the nucleus and the resulting ideal spatial symmetry of the atom, which is an ideal point of the lattice of the diamond [1].

A flat structure of the carbon atom, graphite, results from a structure of the flat nucleus (Fig. 10, b) of the carbon atom (Fig. 11). A comparison of the nuclei (Fig. 10) and the atoms (Fig. 11) of one and the same chemical element, carbon, which is represented in the form of graphite and diamond, explains the causes of their different strength. Laminar graphite (Fig. 11) writes on paper, and diamond, which consists of the utmost symmetric spatial carbon atoms (Fig. 11), cut glass [1].

In Fig. 11, the models of the atoms of graphite, diamond, nitrogen, oxygen and the molecule and the cluster of water resulting from the new theory of microworld, which is based on the Unit-Axiom, are given. There exists the video-world, in which a revolution of the electrons interacting arly with the protons of the atom nuclei is shown

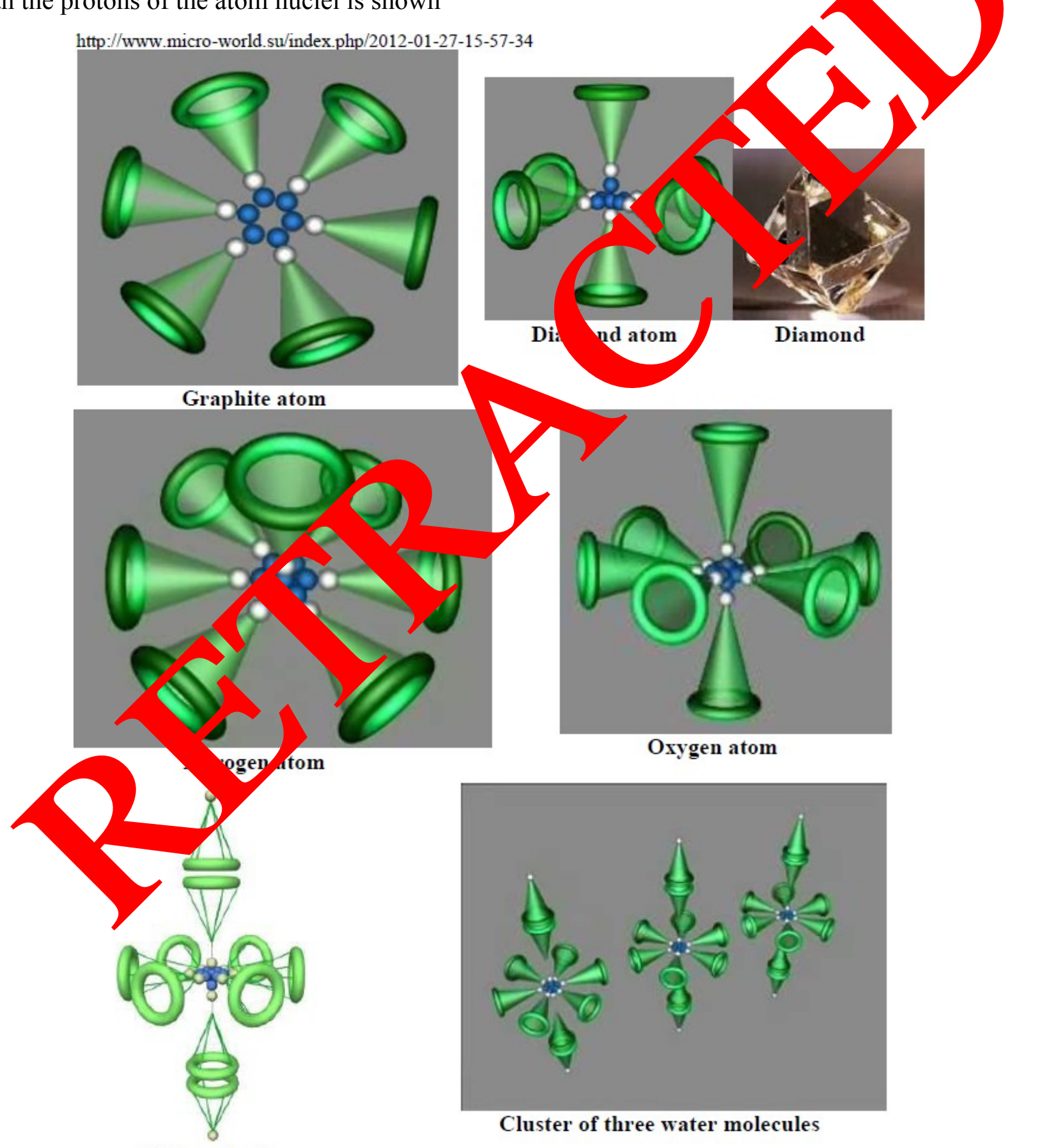

Water molecule

Fig. 11. The nuclei and the atoms of graphite, diamond, nitrogen, oxygen and water molecule and its cluster 
In Figs 12 and 13, one can see the photos of the clusters of graphene and benzene and their theoretical structures resulting from the new theory of microworld, which resolution exceeds the resolution of the modern electron microscopes millionfold (Figs 12, 13) [1].
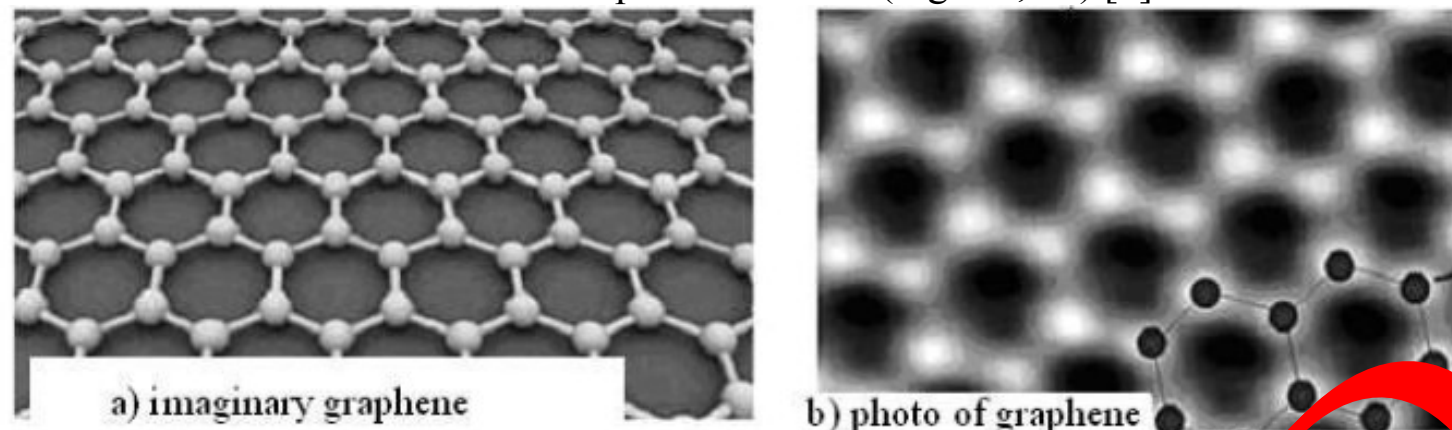

a) imaginary graphene

b) photo of graphene
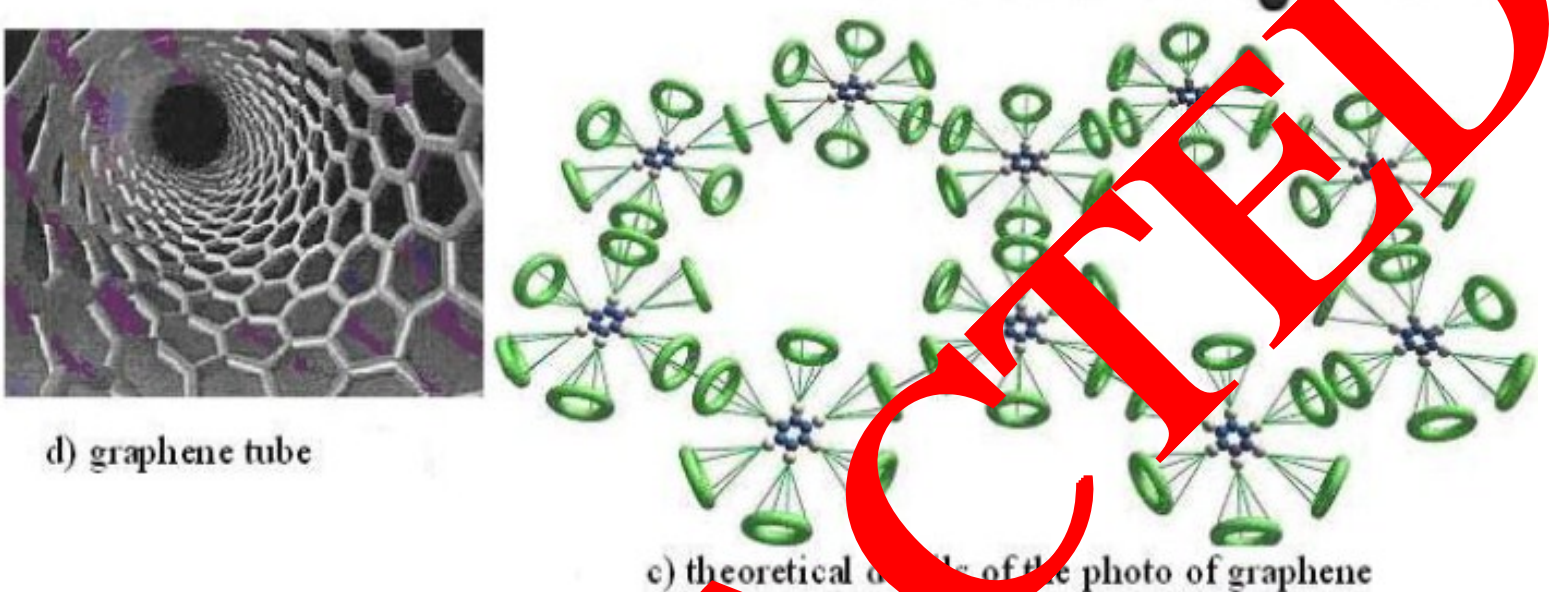

d) graphene tube

c) theoretical o. of $t^{\prime \prime}$ photo of graphene

Fig. 12. Photo of graphene and its theoreti ancture wnere the atomic nucleus structures

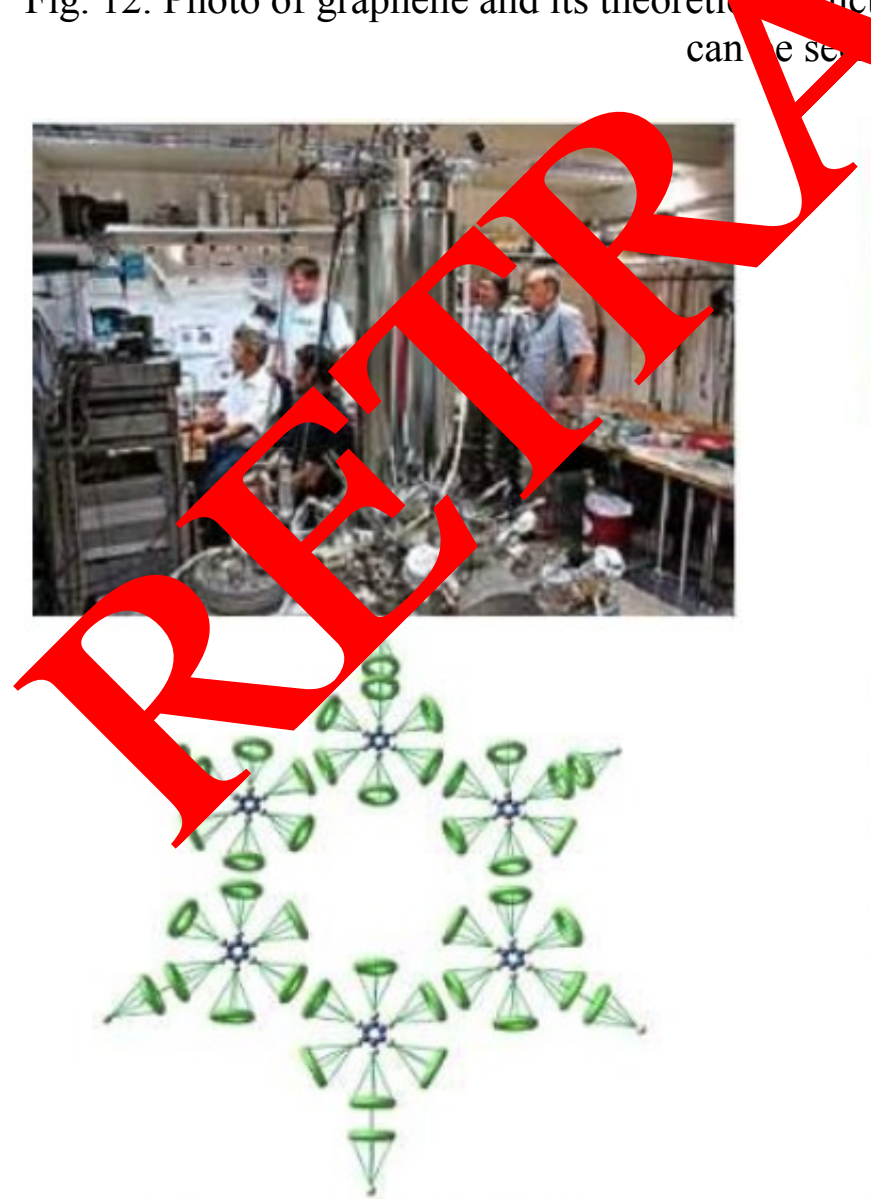

Benzene molecule $\mathrm{C}_{6} \mathrm{H}_{6}$

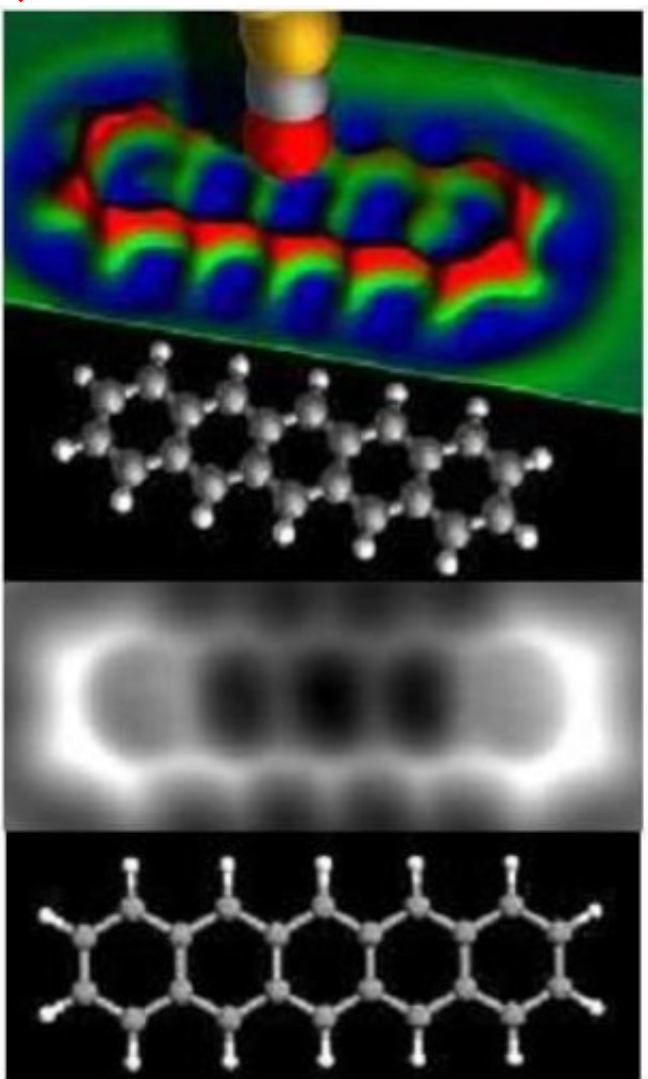

b)

Fig. 13. Leftward: the theoretical structure of benzene; rightward: photo of the benzene clusters 
Let us pay attention to the white spots of the photo of graphene (Fig. 12, b). They are the graphite atoms, which dimensions are nearly $10^{-8} \mathrm{~m}$. The new theory of microworld presents them (Fig. 12, c) in the form of the distinct atoms with the distinct structures of the nuclei, which dimensions are nearly $10^{-15} \mathrm{~m}$. It appears from this that the resolution of the new theory of microworld exceeds the resolution of the modern electron microscopes by $10^{7}$ orders of magnitude (Fig. 12, b) [1].

We are glad to inform that some Russian chemists have already taken interest in the new theory of microworld and have agreed that the electrons have no orbital motions in the atoms. The linear structure of the water molecule (Fig. 14, a) and the angular one (Fig. 14, b) have evoked special interest in them.

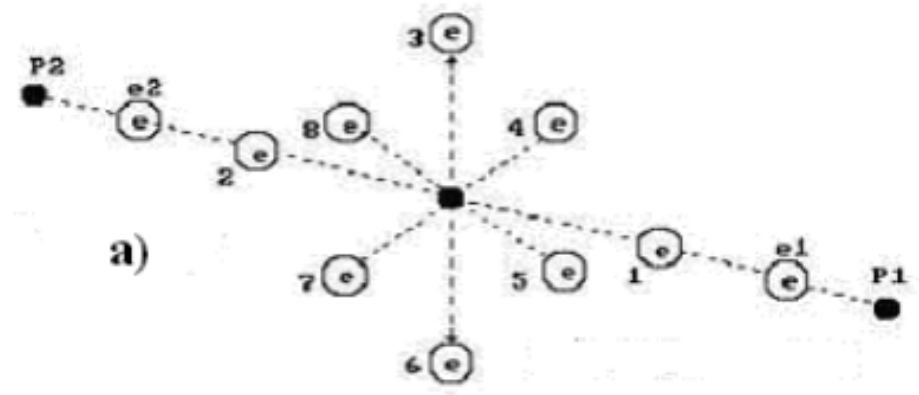

Fig. 14. Structures of the water molecule: a) the linear struc

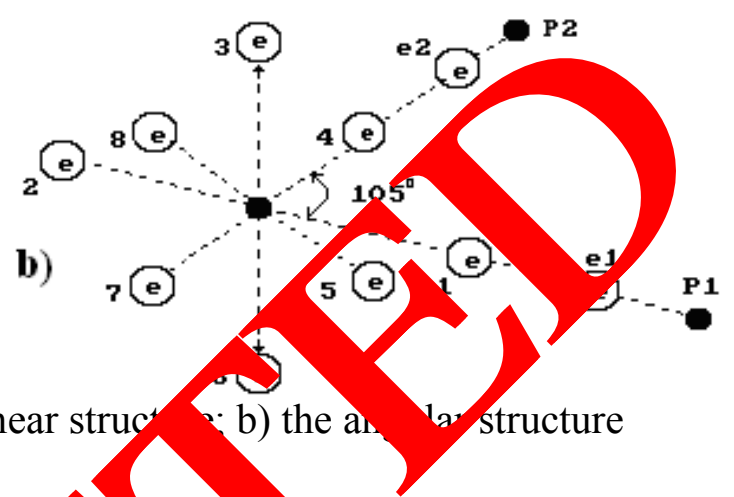

The linear structure of the water molecule (Fig. 14, a) is jo tomente the water crystals in the form of the snowflakes (Fig. 15). The protons (P) of the hydrogen a ms of six linear water molecules join in with six ring electrons of the oxyger atom of the base water molecule and form six-radial structures, which grow and become more a plicated a d form a large variety of the forms of six-radial structures of the crystals and water ch (Fig 15).

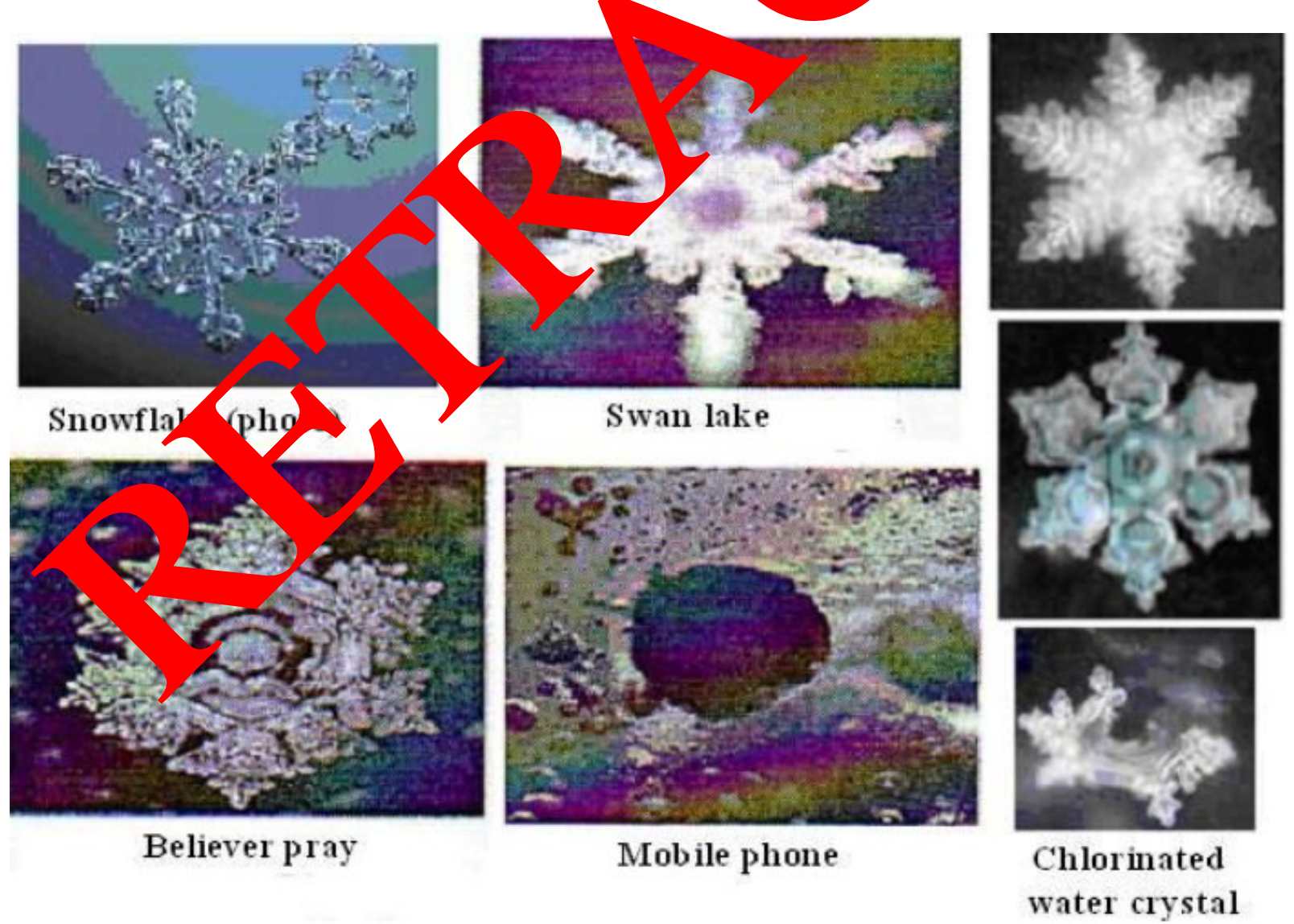

Fig. 15. Crystals of the water molecules: the Russian photo of the snowflake; the Japanese photos, according to them, of the crystals being formed by music, the voice of the prayer and the mobile phone 
Now, let us give the copper atom nucleus structure resulting from the new theory of microworld (Fig. 16 ) [1].

a)

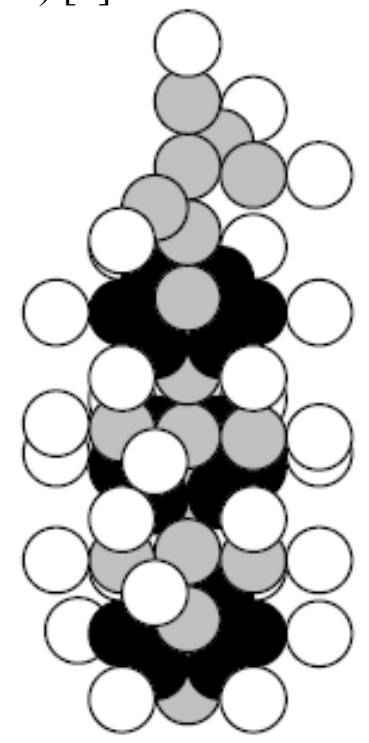

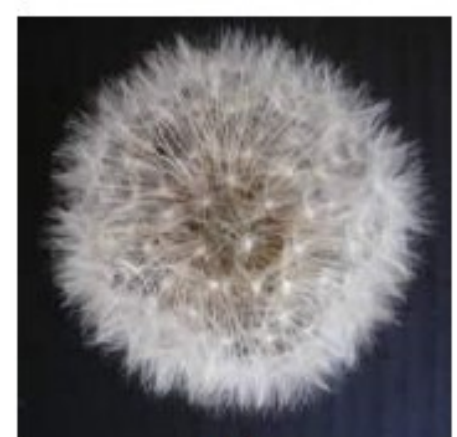

b)

Fig. 16. a) the copper atom nucleas del;

b) possible electronic archite concs of surface of the multiel tron a

Let us pay attention to the fact that the neutrons (the dark bal s an he proton (the bright balls) are arranges in the nucleus according to strict rules, which o und od only in the process of a subsequent construction of the nuclei beginning from se nucleus of hy ogen atom, the simplest
nucleus [1].

\section{CONCLUSION}

The analysis of the microworld inhabitant mod is, result from the old theory of microworld (Figs 1, 3, 4, a and 5) and the mathematical mo els wis describe them, shows almost complete lack of information concerning the restion of $\mathrm{s}$ m the old theory of microworld. All formations resulting from its old theory are illeg e imag $y$ which can be given almost any size. The

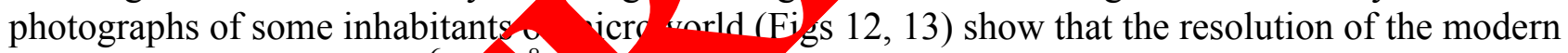
electron microscopes is $10^{-6}$

The new theory of microy reprodu the images at the photos with greater circumstantiation. It presents the nucleus ruc wit the esolution of $10^{-15} \mathrm{~m}$. It means that the resolution of the new theory of micro grd exca the resolution of the modern electron microscopes millionfold minimum.

\section{REFERENCES}

[1].Ph 1. Ka hrev. T Foundations of Physchemistry of Microworld. Monograph. The 15th edition tp:/ mus miero-world.su/ In Russian and in English

http://ww ทicro-world.su/index.php/english

[2]. V.N. D Adenko. Discrete structures of microworld. Publishing house "Knizhny dom LIBROKOM'. M. 2011, 142 pages

[3]. Nikitin A.N. Structure of a kernel and electronic covers of atoms. http://atom21.ru/ 